\title{
Tuberous sclerosis presenting with fetal and neonatal cardiac tumours
}

\author{
G Wallace, H C Smith, G H Watson, S Rimmer, S W D'Souza
}

\begin{abstract}
Cardiac tumours were identified on ultrasonography in fetal or early postnatal life in five infants. Tuberous sclerosis was subsequently diagnosed in all five. Only one infant required operation. Regression of the tumour occurred in three. No infant had hypomelanotic macules at birth, and they took up to two years to appear.
\end{abstract}

Cardiac tumours are a common feature of tuberous sclerosis, but they are rarely diagnosed in life unless they are causing obstructive symptoms or arrhythmias. Echocardiographic studies, however, have shown tumours in over half of affected patients, none of whom had cardiac symptoms. ${ }^{1-3}$

Cardiac tumours have not been described as the sole sign of tuberous sclerosis in adults, ${ }^{4}$ but our experience with fetal and neonatal cardiac tumours shows that they may precede the skin and neuroradiological signs of the disease.

\section{Case reports}

CASE 1

A 5 day old baby was referred for a cardiologist's opinion because a murmur had been noted on routine examination. He had no symptoms and his electrocardiogram was normal. Echocardiography showed multiple cardiac tumours, the largest of which was partially obstructing the left ventricular outflow tract. Serial echocardiograms showed spontaneous regression of the tumours so that when last seen (at the age of 5 years) only one tumour $8 \mathrm{~mm}$ in diameter remained in the right ventricle. Illustrations of regression of the tumours in this child have previously been reported by Smith $e t a l$ in their figures $2-6 .^{3}$

Careful examination of the skin, and computed tomography scans of the brain, were carried out at 2 and 5 months but failed to show any signs of tuberous sclerosis. By the age of 12 months, however, a streak of depigmented scalp hair and a depigmented macule $3 \mathrm{~mm}$ in diameter had appeared. Further depigmented skin patches appeared over the following year. Atypical absence seizures developed at the age of 2 years.

Both parents were investigated by careful skin and fundoscopic examination, computed tomography, radiographs of the hands, and cardiac ultrasonography. No signs of tuberous sclerosis were found. The child's father has Wolff-Parkinson-White syndrome, and the mother has facial acne rosacea.
CASE 2

A healthy 21 year old mother had a normal pregnancy until 34 weeks' gestation, when an irregular fetal bradycardia was noted. Ultrasonography of the fetal heart was normal at 34 weeks, but a further scan a week later showed echodense areas in the right ventricle that persisted until delivery.

During the neonatal period the baby continued to have episodes of bradycardia and multifocal ectopic beats. Cardiac ultrasonography showed three intracardiac masses in the right ventricle. Cardiac catheterisation confirmed the presence of the tumours, and also showed irregularity of the ventricular septum suggestive of additional pea sized tumours within the septum. Over the next two years serial ultrasound scans showed regression of all the tumours to minute proportions.

Cranial ultrasonography at the age of 7 days suggested some increased echodensity in the left frontal region. Renal ultrasonography, and examination of the skin with a Wood's light gave normal results. Computed tomography and magnetic resonance imaging at the age of 7 weeks showed two areas of decreased attenuation in the frontal horns that were similar to the changes of resolving infarction. Despite further examination with the Wood's light, no depigmented patches were found until the age of 2 years when seizures and facial angiofibromas developed, and periventricular calcification was seen on computed tomography.

Both parents were examined for evidence of tuberous sclerosis; there were no cutaneous stigmata, and results of computed tomograms, renal ultrasound scans, and examination of the fundi were normal.

CASE 3

A 22 year old woman with tuberous sclerosis was scanned routinely during her first pregnancy. Fetal cardiac tumours were first identified in the right and left ventricles at 28 weeks' gestation. The pregnancy proceeded uneventfully to normal delivery at $\mathbf{4 2}$ weeks. The baby was symptom free at birth, and postnatal echocardiography confirmed the presence of ventricular tumours measuring $10 \times 10 \mathrm{~mm}$ on the left, and $10 \times 8 \mathrm{~mm}$ on the right. Serial scans showed the tumours becoming progressively less dense during the first 12 months of life.

Examination of the skin in the newborn period failed to show any other signs of tuberous sclerosis. At the age of 11 weeks the baby had a focal seizure. A computed tomogram 
showed a number of calcified periventricular nodules that were characteristic of tuberous sclerosis, but examination of the skin showed no abnormality. Depigmented skin patches did not appear until the age of 14 months, and by 17 months he was developing facial angiofibromas.

The mother underwent echocardiography postnatally and two tumours, each $5 \mathrm{~mm}$ in diameter, were found in her left ventricle.

CASE 4

The mother of case 3 had a second pregnancy in 1988. Antenatal scan at 32 weeks' gestation showed multiple small echogenic areas in the lateral wall of the fetal left ventricle, the interventricular septum, and in the right ventricle near the tricuspid valve. The pregnancy proceeded uneventfully to normal delivery at full term.

Postnatal echocardiography confirmed the antenatal findings. Cranial ultrasonography and examination of the skin with a Wood's light showed no abnormality. Mother and baby were discharged after 48 hours, and the baby remained symptom free. At the age of 5 months, two depigmented macules appeared on the chest and left knee. When last seen at the age of 18 months his speech and motor development were normal.

\section{CASE 5}

A 17 year old mother underwent a routine scan at 20 weeks' gestation in her first pregnancy. A single large tumour was identified in the fetal right ventricular outflow tract. At birth the baby was well and examination of the skin with a Wood's light, and cranial ultrasonography, failed to show any additional features of tuberous sclerosis. Possible seizures at 4 months prompted computed tomography of the brain, which showed multifocal periventricular calcification. A month later, because of potential outflow tract obstruction, the baby underwent resection of a solitary cardiac tumour, which histological examination showed to be a rhabdomyoma. When last seen at the age of 18 months, examination of the skin showed no abnormality, speech development was delayed, and he had focal and generalised seizures that required treatment with valproate and nitrazepam.

His mother's medical history was reviewed. She had developed papules on her cheeks at the age of 6 years and had consulted a dermatologist, but not been given a diagnosis. She had had bilateral spontaneous pneumothoraces during pregnancy.

On further investigation, multiple echogenic lesions characteristic of angiolipomas were seen on renal ultrasonography, and there were cerebral changes consistent with tuberous sclerosis on her computed tomogram. Examination of the skin with a Woods light did not show any depigmentation.

\section{Discussion}

Tuberous sclerosis is an autosomal dominant condition with high penetrance, and an estimated prevalence between $1 / 15000$ and 1/30 000 . There is a slight male preponderance, and 50 to $80 \%$ of those affected seem to have resulted from new mutation. ${ }^{5}$

Cardiac rhabdomyomas are among the earliest signs of tuberous sclerosis and occur in over half of affected patients. ${ }^{1-3}$ By contrast, primary cardiac tumours are rare in the general population, and extremely uncommon in childhood. ${ }^{6}$ Necropsy studies have suggested that $37-50 \%$ of those dying of cardiac rhabdomyomas have tuberous sclerosis. ${ }^{78}$ This is almost certainly an underestimate because the studies include a high proportion of stillborn infants and neonates, who would not necessarily show any other diagnostic features.

In the present series the cardiac ultrasound appearances were characteristic of rhabdomyoma, and all the infants subsequently developed other signs of tuberous sclerosis. Cases 1 and 2 illustrate the difficulty in establishing a firm diagnosis of tuberous sclerosis despite extensive investigation and keeping the possibility in mind. It is probable that most infants with cardiac rhabdomyomas have tuberous sclerosis, ${ }^{9}$ and this diagnosis must not be discounted because the initial examination of the skin and computed tomograms give normal or atypical results. The areas of decreased attenuation on computed tomography in case 2 probably represented areas of demyelination rather than infarction, but it is unusual to find these without periventricular nodules. ${ }^{10}$ Initial results of magnetic resonance imaging in tuberous sclerosis suggest that this may be a more sensitive method of detecting early changes ( $M$ Gomez. Presented at the Tuberous Sclerosis Association Symposium, 1988). Cranial ultrasonography gave abnormal results in one of the three cases scanned in the neonatal period, and its place in the diagnosis of tuberous sclerosis requires further evaluation.

The cardiac tumour detected at 20 weeks' gestation in case 5 is the earliest recorded instance of a fetal tumour associated with tuberous sclerosis. Tumours in the fetuses studied increased in both size and number towards full term, suggesting that maternal factors influence their growth. The only infant with symptoms in this series developed arrhythmias before birth, and before tumours were recognised. Postnatal tumour regression has so far been recorded in three of the five infants, and this also seems to be an active process and not merely the effect of growth of the cardiac chambers.

Despite the usually benign nature of rhabdomyomas, they may cause death from mechanical obstruction or disturbance of rhythm. In the largest necropsy series, $70 \%$ of the deaths occurred in the first year and the authors recommended that patients with symptoms should have operations. ${ }^{9}$ The availability of ultrasonography permits detection of cardiac tumours in fetal and early neonatal life, and raises questions about appropriate management. There are case reports of successful resection after the prenatal detection of tumours, ${ }^{11}{ }^{12}$ but as tumour regression is now well documented 
we strongly recommend a conservative approach, reserving operation for those infants with life threatening disturbances of rhythm or outflow tract obstruction by a solitary tumour. ${ }^{3} 13$

Antenatal detection of fetal cardiac tumours should prompt careful examination of parents and siblings for signs of tuberous sclerosis, whether or not this diagnosis is established in the baby. Most authors recommend detailed skin examination with a Wood's light, fundoscopy, renal ultrasonography, and computed tomography of the brain. Even though prevalence of the tumour is low in adults, ${ }^{3}$ we include echocardiography, as cardiac tumours are an important marker of the condition. Termination of pregnancy has been carried out after the detection of cardiac tumours in the fetus of an affected mother. ${ }^{14-16}$

Tuberous sclerosis shows pronounced variation in phenotypic expression, and the characteristic skin lesions and computed tomographic signs are often absent at birth. Hypomelanotic macules eventually appear in $90 \%$ of those with tuberous sclerosis, and have been regarded as the earliest sign of the condition. Unfortunately the sign is not specific to tuberous sclerosis, as 2-3/1000 normal newborn infants will also have one or two such patches. Late appearance of hypomelanotic macules in tuberous sclerosis is recognised, ${ }^{17}$ and in this series none was identified in the neonatal period despite careful examination with a Wood's light. Four of the five children went on to develop hypomelanotic macules, although they took from five months to two years to appear.

We suggest that most fetal or neonatal cardiac tumours are diagnostic of tuberous sclerosis. Multiple ventricular tumours are highly likely to be associated with tuberous sclerosis, ${ }^{79}$ and the diagnosis should be considered even when a single tumour is detected, as a quarter of the tumours found on ultrasound screening of adults and children with tuberous sclerosis are solitary. ${ }^{3}$ As cardiac tumours may remain the sole sign of the condition for many months, families should be offered a presumptive diagnosis and genetic counselling even when other signs of tuberous sclerosis are absent.

The authors thank Dr YDL Sibley, Dr J Wright, and Dr DG Sims who referred three of the children, and Dr M Super for his comments on the text.

1 Gibbs JL. The heart and tuberous sclerosis. Br Heart $\mathcal{f}$ 1985;54:596-6.

2 Bass JL, Breningstall GN, Swaiman KF. Echocardiographic incidence of cardiac rhabdomyoma in tuberous sclerosis. Am $\mathcal{f}$ Cardiol 1985;55:1379-82.

3 Smith HC, Watson GH, Patel RG, Super M. Cardiac rhabdomyomata in tuberous sclerosis: their course and rhabdomyomata in tuberous sclerosis: their cou
diagnostic value. Arch Dis Child 1989;64:196-200.

4 Fryer AE, Chalmers A, Connor JM, et al. Evidence that the gene for tuberous sclerosis is on chromosome 9. Lancet $1987 ; \mathrm{i}: 659-60$

5 Hunt A, Lindenbaum RH. Tuberous sclerosis: a new estimate of prevalence within the Oxford region. $\mathcal{f} \mathrm{Med}$

6 Arcnet 1984;21:272-7. EW. Primary cardiac tumours in children. $\mathcal{F}$ Thorac Cardiovasc Surg 1980;79:582-91.

7 Fenoglio JJ, McAllister HA, Ferrans VJ. Cardiac rhabdomyoma: a clinicopathologic and electron microscopic study. Am F Cardiol 1976;38:241-51.

8 Batchelor TM, Maun ME. Congenital glycogenic tumours of the heart. Archives of Pathology 1945;39:67-73.

9 Pomerance A, Davies MJ. The pathology of the heart. Oxford: Blackwell, 1975.

10 Kingsley DPE, Kendall BE, Fitz CR. Tuberous sclerosis: a clinicoradiological evaluation of 110 cases with particular reference to atypical presentation. Neuroradiology 1986;28:

11 Di Lollo L, Castellani R, Maiorana A. Ultrasonic patterns of a cardiac rhabdomyoma detected in utero. $\mathcal{F}$ Perinat Med

12 Pesonen E, Leijala M, Jarvenpaa A-L, Teramo K. Cardiac rhabdomyomas in a newborn baby: early diagnosis and treatment. Acta Paediatr Scand 1985;74:824-7.

13 Stijns $\mathbf{M}$, Linteermans J, Tremournoux $\mathbf{M}$, Vliers $\mathbf{A}$. Spontaneous disappearance of aortic subvalvular obstruction in infants with tuberous sclerosis. Pediatr Cardiol 1982;3: 88-9.

14 Crawford DC, Garrett C, Tynan M, Neville BG, Allan LD. Cardiac rhabdomyomata as a marker for the antenatal detection of TS. F Med Genet 1983;20:303-12.

15 Platt LD, Devore GR, Horenstein J, Pavlova Z, Kovacs B Falk RE. Prenatal diagnosis of TS: the use of fetal echocardiography. Prenat Diagn 1987;7:407-11.

16 Muller L, De Jong G, Falck V, Hewlett R, Hunter J, Shires $\mathrm{J}$. Antenatal ultrasonographic findings in tuberous sclerosis. $S$ Afr Med $\mathcal{~}$ 1986;69:633-8.

17 Oppenheimer EY, Rosman NP, Dooling EC. The late appearance of hypopigmented maculae in tuberous sclerosis. 